\title{
Emergence of Escherichia coli producing OXA-48 $\beta$-lactamase in the community in Switzerland
}

\author{
Katrin Zurfluh', Magdalena T Nüesch-Inderbinen ${ }^{1}$, Laurent Poirel ${ }^{2}$, Patrice Nordmann², Herbert Hächler ${ }^{1}$ \\ and Roger Stephan ${ }^{1 *}$
}

\begin{abstract}
Background: The emergence and worldwide spread of carbapenemase-producing Enterobacteriaceae is of great concern to public health services. The aim of this study was to investigate the occurrence of carbapenemase-producing Enterobacteriaceae in the community in Switzerland.

Findings: One thousand and eighty-six stool samples of healthy humans (staff members of a food-processing company which were screened for the occurrence of salmonellae) were collected in September 2014. After an initial enrichment-step, carbapenemase-producing Enterobacteriaceae were isolated from the carbapenem-containing selective medium SUPERCARBA II. Grown colonies from 11 samples were screened by PCR for the presence of bla $a_{\mathrm{KPC}}$ bla $a_{\mathrm{NDM}}, b_{a_{\mathrm{OXA}}-48}$ and bla $\mathrm{VIM}$. A single OXA-48-producing Escherichia coli was detected. Phylogenetic grouping and multi-locus sequence typing (MLST) revealed that this strain belonged to D:ST38, a type which had been previously reported in the UK, France, Lebanon and Egypt.

Conclusions: The results of this study show that OXA-48-producing Enterobacteriaceae have started to spread into the community in Switzerland, and a continuous monitoring is necessary to better understand their dissemination in the human population as well as in animals and the environment.
\end{abstract}

Keywords: Carbapenemase, Community, Enterobacteriaceae, Dissemination, OXA-48

\section{Findings}

Most carbapenemases identified in Enterobacteriaceae hydrolyze almost all $\beta$-lactam antibiotics, including carbapenems, with the exception of OXA-48 that weakly hydrolyses carbapenems but paradoxally spares broadspectrum cephalosporins. Currently, the most prevalent carbapenemases found among Enterobacteriaceae worldwide include the Ambler class A carbapenemase KPC, the class B metallo- $\beta$-lactamases (MBLs) of the IMP-, NDM- or VIM-type, and the class D OXA-48-like oxacillinases [1].

Carbapenemase-producing Enterobacteriaceae (mainly Klebsiella pneumoniae and Escherichia coli) were first described in Europe in the 1990s, and since then they have been increasingly reported especially in clinical settings. The emergence of these bacteria is of great concern to public health services because they are often also

\footnotetext{
* Correspondence: stephanr@fsafety.uzh.ch

'Institute for Food Safety and Hygiene, Vetsuisse Faculty University of Zurich, Winterthurerstrasse 272, 8057 Zurich, Switzerland

Full list of author information is available at the end of the article
}

co-resistant to many other antibiotic classes and thus, only few treatment options remain [2]. Their dissemination into the community and the environment is only a matter of time.

Two years ago, we reported that there was no evidence for the dissemination of carbapenemase-producing Enterobacteriaceae in the community in Switzerland [3]. The aim of this study was to survey the current situation for the presence of carbapenemase-producing Enterobacteriaceae in healthy humans in Switzerland.

In the context of a yearly routine fecal screening for salmonellae, stool samples were obtained from 3200 employees of a meat-processing company. This company consists of eight countrywide processing plants and employs people from the surrounding urban areas. Each staff member was tested only once. For this study, 1086 samples were randomly selected. The samples were tested for the presence of KPC-, NDM-, OXA-48, or VIMtype carbapenemase-producing Enterobacteriaceae. A loopful of each sample was enriched for 24 hours at $37^{\circ} \mathrm{C}$ in $5 \mathrm{~mL}$ of Enterobacteriaceae Enrichment (EE) broth 
without antibiotic (Oxoid, Ltd, Basingstoke, Hampshire, England). The enrichment culture was thereafter streaked onto a modified SUPERCARBA agar medium selecting for bacteria with reduced susceptibility to ertapenem, which is the most sensitive marker for carbapenemase production. The SUPERCARBA II medium differs from the previously published SUPERCARBA [4] since its basis is made of Trypticase Soy agar (rather than Drigalski) and contains vancomycin $(10 \mu \mathrm{g} / \mathrm{ml})$ and amphotericin B $(5 \mu \mathrm{g} / \mathrm{ml})$ in order to prevent the growth of Gram positives or fungi. SUPERCARBA, ideally complemented by the RAPIDEC ${ }^{\circ}$ CARBA NP test (bioMérieux Ltd), has been shown to be most suited to detect carbapenemase producers, since it exhibits a very high specificity and a very high sensitivity even for those OXA48-producing strains that often exhibit low MICs of carbapenems [4].

Growth of oxidase negative colonies was obtained for eleven selective plates and those colonies were screened by PCR for the presence of $b l a_{\mathrm{KPC}}, b l a_{\mathrm{NDM}}, b l a_{\mathrm{OXA}-48}$ and $b l a_{\mathrm{VIM}}$ using primers described previously $[5,6]$. Species identification using API ID 32 E (bioMérieux, Marcy l'Etoile, France) and multi-locus sequence typing [7] was performed with strains positive for any of the above listed genes. Phylogenetic classification was performed as described previously [8].

A single isolate (JU-S-791) was found positive for the bla $a_{\text {OXA-48 }}$ carbapenemase gene (100\% identity after sequencing), which corresponded to a shedding prevalence of carbapenemase-producing Enterobacteriaceae in the tested collective of $0.09 \%$. The Oxa-48-positive individual, a Turkish citizen born and raised in Switzerland, had no history of hospital admissions and no history of recent antibiotic therapy but reported annual visits to Turkey and a journey to Italy one month prior to sampling. The OXA-48 producer was identified as E. coli sequence type (ST) 38 and belonged to the extraintestinal pathogenic phylogenetic group D.

Susceptibility testing was performed by the agar diffusion method, using antibiotic disks (Becton Dickinson and Company, Maryland, USA) according to the manufacturers' protocols. Minimal inhibitory concentrations (MIC) of imipenem and cefotaxime, ceftazidime, and cefepime alone and in combination with clavulanic acid were determined by Etest strips (bioMérieux, Marcy l'Etoile, France). Results were interpreted according to the criteria of the Clinical and Laboratory Standards Institute (CLSI) [9].

The MIC of imipenem was $0.5 \mu \mathrm{g} / \mathrm{ml}$, which is still below the susceptibility breakpoint defined by CLSI. Class D carbapenem-hydrolyzing $\beta$-lactamases possess weak carbapenemase-activity and usually do not confer a high-level resistance phenotype unless the strain exhibits additional permeability defects $[10,11]$.
Although OXA-48-producers only very poorly hydrolyze expanded-spectrum cephalosporins, the strain JU-S-791 displayed an extended-spectrum $\beta$-lactamase (ESBL) phenotype (MIC of cefotaxime $>16 \mu \mathrm{g} / \mathrm{ml}$, and MIC of cefotaxime plus clavulanic acid $=0.5 \mu \mathrm{g} / \mathrm{ml}$ ) and additionally tested resistant in the disk diffusion test to gentamicin (zone diameter $10 \mathrm{~mm}$ ), nalidixic acid, $(6.5 \mathrm{~mm})$, sulfamethoxazole $(6 \mathrm{~mm})$ and trimethoprim $(6 \mathrm{~mm})$. It was susceptible in the disk diffusion test to ciprofloxacin $(27 \mathrm{~mm})$, tetracycline $(19 \mathrm{~mm})$, chloramphenicol $(25 \mathrm{~mm})$ and kanamycin $(19 \mathrm{~mm})$. Screening for $b l a_{\mathrm{CTX}-\mathrm{M}}, b l a_{\mathrm{SHV}}$ and $b l a_{\mathrm{TEM}}$ as described previously [12], revealed that the strain, in addition to the bla OxA48 gene, co-harbored the ESBL gene $b l a_{\mathrm{CTX}-\mathrm{M}-24}$ and the narrow-spectrum $\beta$-lactamase gene $b l a_{\mathrm{TEM}-1}$. In order to evaluate whether the bla $a_{\mathrm{OXA}-48}$ gene could be transferred, conjugation experiments were performed using a standardized method for liquid mating techniques, as described elsewhere [13]. However, they systematically failed, suggesting a chromosomal location of this gene, and no further attempts at plasmid detection were undertaken.

OXA-48 was first described 2001 in a K. pneumoniae isolate from a patient in Istanbul, Turkey. It is suggested that the main reservoirs of OXA-48-harbouring $K$. pneumoniae and E. coli are in North African countries and Turkey [11]. Nowadays, OXA-48 producing Enterobacteriaceae are widely disseminated throughout many European countries and have led to several hospital outbreaks [11]. In particular, the spread of the bla $a_{\text {OXA-48 }}$ gene in community isolates but also in the environment has been demonstrated in Morocco [14,15]. The successful spread of $b l a_{\text {OXA-48 }}$ is linked to a single self-conjugative $62 \mathrm{~kb}$ IncL/ $\mathrm{M}$ plasmid which was so far only identified in Enterobacteriaceae [16]. More recently, a chromosomal location of $b l a_{\mathrm{OXA}-48}$ was reported from isolates in the UK, France, Egypt, Lebanon and Switzerland [17-19], not only among humans but also in fowl [20]. Interestingly, chromosomal location of bla $a_{\mathrm{OXA}-48}$ was mostly associated with $E$. coli D: ST38 co-harboring $b l a_{\mathrm{CTX}-\mathrm{M}-24}$ and $b l a_{\mathrm{TEM}-1}-$ the same strain type described in this study. Considering these similarities, it is highly probable that the strain described in this study also contains a chromosomally encoded $b l a_{\text {OXA-48. }}$.

In conclusion, our study demonstrates that the prevalence of carbapenemase-producing Enterobacteriaceae in healthy humans in Switzerland is $0.09 \%$ (95\% CI $=<0.0001-0.0057$ ) and is associated with E. coli D:ST38. This particular strain has previously been predominantly associated with clinical settings $[18,19,21]$. Our results strongly suggest that this epidemic clone, harboring a non-transmissible $b l a_{\text {OXA-48 }}$ gene, is currently circulating among healthy humans. Notably, this further demonstrates that the problem of the dissemination of bla $a_{\text {OXA-48 }}$ positive isolates is not only related to the epidemicity of the widely identified plasmid pOXA- 
48a [22] among different strain backgrounds, but includes the dissemination of a single E. coli ST38 strain harboring the $b l a_{\mathrm{OXA}-48}$ gene on its chromosome. It is now even more important that further studies are carried out in order to survey the dissemination of carbapenemase-producing enterobacterial strains in the population. As a strategy for ensuring the detection of strains that exhibit low MICs of carbapenems such as OXA-48-producers, the use of the SUPERCARBA II may be considered highly recommendable.

\section{Competing interests}

An international patent form for the Supercarba medium has been filed on behalf of INSERM Transfert (Paris, France) involving LP as PN as COdiscoverers.

\section{Authors' contributions}

$\mathrm{RS}, \mathrm{LP}, \mathrm{HH}$ and $\mathrm{MNI}$ designed the study. KZ drafted the manuscript and carried out the microbiological and molecular biological tests. All authors read and approved the final manuscript.

\section{Acknowledgements}

This project was partially funded by the Swiss Federal Office of Public Health.

\section{Author details}

'Institute for Food Safety and Hygiene, Vetsuisse Faculty University of Zurich, Winterthurerstrasse 272, 8057 Zurich, Switzerland. ${ }^{2}$ Medical and Molecular Microbiology Unit "Emerging Antibiotic Resistance", Dept of Medicine, Faculty of Science, University of Fribourg, Fribourg, Switzerland.

Received: 21 January 2015 Accepted: 16 March 2015

Published online: 26 March 2015

\section{References}

1. Cantón R, Akóva M, Carmeli Y, Giske CG, Glupczynski Y, Gniadowski M, et al. Rapid evolution and spread of carbapenemases among Enterobacteriaceae in Europe. Clin Microbiol Infect. 2012;18:413-31.

2. Grundmann H, Livermore DM, Giske CG, Canton R, Rossolini GM, Campos J, et al. Carbapenem-non-susceptible Enterobacteriaceae in Europe: conclusions from a meeting of national experts. Euro Surveill. 2010;15:1-13.

3. Nüesch-Inderbinen M, Zurfluh K, Hächler H, Stephan R. No evidence so far for the dissemination of carbapenemase-producing Enterobactericeae in the community in Switzerland. Antimicrob Resist Infect Control. 2013;2:23.

4. Nordmann P, Girlich D, Poirel L. Detection of carbapenemase producers in Enterobacteriaceae by use of a novel screening medium. J Clin Microbiol. 2012;50:2761-6.

5. Ellington MJ, Kistler J, Livermore DM, Woodford N. Multiplex PCR for rapid detection of genes encoding acquired metallo-beta-lactamases. J Antimicrob Chemother. 2007:59:321-2.

6. Poirel L, Walsh TR, Cuvillier V, Nordmann P. Multiplex PCR for detection of acquired carbapenemase genes. Diagn Microbiol Infect Dis. 2011;70:119-23.

7. Wirth T, Falush D, Lan R, Colles F, Mensa P, Wieler LH, et al. Sex and virulence in Escherichia coli: an evolutionary perspective. Mol Microbiol. 2006;60:1136-51

8. Clermont $\mathrm{O}$, Bonacorsi $\mathrm{S}$, Bingen E. Rapid and simple determination of the Escherichia coli phylogenetic group. Appl Env Microbiol. 2000;66:4555-8.

9. Clinical and Laboratory Standarts Institute. Performance Standards for Antimicrobial Susceptibility Testing: Twenty-First Informational Supplement. CLSI document M100-S23. Wayne, PA 19087 USA: The Clinical and Laboratory Standarts Institute; 2013.

10. Evans BA, Amyes SGB. OXA $\beta$-lactamases. Clin Microbiol Rev. 2014;27:241-63.

11. Nordmann P, Poirel L. The difficult-to-control spread of carbapenemase producers among Enterobacteriaceae worldwide. Clin Microbiol Infect. 2014;20:821-30.

12. Geser N, Stephan R, Hächler H. Occurrence and characteristics of extended-spectrum $\beta$-lactamase (ESBL) producing Enterobacteriaceae in food producing animals, minced meat and raw milk. BMC Vet Res. 2012;8:21.

13. Zurfluh $K$, Jakobi G, Stephan R, Hächler H, Nüesch-Inderbinen M. Replicon typing of plasmids carrying bla CTX-M-1 $_{\text {in }}$ in Enterobacteriaceae of animal, environmental and human origin. Front Microbiol. 2014;5:555.

14. Potron A, Poirel L, Bussy F, Nordmann P. Occurrence of the carbapenem-hydrolyzing $\beta$-lactamase gene bla OXA-48 $_{48}$ in the environment in Morocco. Antimicrob Agents Chemother. 2011;55:5413-4.

15. Girlich D, Bouihat N, Poirel L, Benouda A, Nordmann P. High rate of faecal carriage of extended-spectrum $\beta$-lactamase and OXA-48 carbapenemase-producing Enterobacteriaceae at a university hospital in Morocco. Clin Microbiol Infect. 2014;20:350-4.

16. Poirel L, Potron A, Nordmann P. OXA-48-like carbapenemases: the phantom menace. J Antimicrob Chemother. 2012;67:1597-606.

17. Potron A, Poirel L, Rondinaud E, Nordmann P: Intercontinental spread of OXA-48 beta-lactamase-producing Enterobacteriaceae over a 11-year period, 2001 to 2011. Euro Surveill. 2013;18(31). (http://www.eurosurveillance.org/ ViewArticle.aspx?Articleld=20549)

18. Dimou V, Dhanji H, Pike R, Livermore DM, Woodford N. Characterization of Enterobacteriaceae producing OXA-48-like carbapenemases in the UK. J Antimicrob Chemother. 2012;67:1660-5.

19. Beyrouthy R, Robin F, Dabboussi F, Mallat H, Hamzé M, Bonnet R. Carbapenemase and virulence factors of Enterobacteriaceae in North Lebanon between 2008 and 2012: evolution via endemic spread of OXA-48. J Antimicrob Chemother. 2014;69:2699-705.

20. Al Bayssari C, Olaitan AO, Dabboussi F, Hamze M, Rolain JM. Emergence of OXA-48-producing Escherichia coli clone ST38 in fowl. Antimicrob Agents Chemother. 2015;59:745-6

21. Poirel L, Bernabeu S, Fortineau N, Podglajen I, Lawrence C, Nordmann P. Emergence of OXA-48-producing Escherichia coli clone ST38 in France. Antimicrob Agents Chemother. 2011;55:4937-8.

22. Potron A, Poirel L, Nordmann P. Derepressed transfer properties leading to the efficient spread of the plasmid encoding carbapenemase OXA-48. Antimicrob Agents Chemother. 2014;58:467-71.

\section{Submit your next manuscript to BioMed Central and take full advantage of:}

- Convenient online submission

- Thorough peer review

- No space constraints or color figure charges

- Immediate publication on acceptance

- Inclusion in PubMed, CAS, Scopus and Google Scholar

- Research which is freely available for redistribution 